\title{
BETATRON TUNE SPREAD GENERATION AND DIFFERENTIAL CHROMATICITY CONTROL BY OCTUPOLE FAMILIES AT TEVATRON*
}

\author{
P. M. Ivanov", Y. Alexahin, J. Annala, V. A. Lebedev, FNAL, Batavia, IL, 60510, USA
}

\section{Abstract}

Existing Tevatron octupoles have been rearranged into four functional families. Two of these families generate betatron tune spreads in the vertical and horizontal planes whereas the other two control the differential chromaticity between the proton and antiproton helices. The calculated effect on the tunes and chromaticity is compared with direct measurements. Analytical formulas for betatron tune distribution functions are presented.

\section{INTRODUCTION}

In the past, the head-tail instability in Tevatron was suppressed by large machine chromaticities. This amplified the beam-beam effects and poorly affected the beam lifetime. Recently, to prevent the instability at zero chromaticities, the octupoles have been introduced into machine operation. Consequently, the beam lifetime during injection is significantly improved. The beam stabilization comes from Landau damping induced by the octupole-generated tune spread consisting of two contributions. One of them comes from the betatron tune dependence on transverse amplitudes and the second from the quadratic chromaticity excited by octupoles positioned at locations with non-zero dispersion. To separate the proton and antiproton beams during injection the electrostatic separators are used to move them to antisymmetric helical orbits with a radius of about $10 \mathrm{~mm}$. This sufficiently large helix results in changes of lattice parameters when the octupoles are turned on. The octupole families were designed to minimize these undesirable effects using computer simulation with the experimentally verified Tevatron lattice model.

\section{OCTUPOLE FAMILY ARRANGEMENT}

Existing machine octupoles [1] have been connected into four new functional families. The families were designed to insure their maximum effectiveness and to avoid the dynamic aperture loss due to non-linearities. Two families, OF (12 octupoles, $\beta_{x}>\beta_{y}$ ) and OD (18 octupoles, $\beta_{x}<\beta_{y}$ ), are assigned to excite the betatron tune spreads in the vertical and horizontal planes. Both octupole families excite quadratic chromaticities, which make additional contribution to the betatron tune spreads. Two other octupole families, 01 (14 octupoles, $\beta_{x}>\beta_{y}$ ) and $\mathbf{O 2}$ (18 octupoles, $\beta_{x}<\beta_{y}$ ), together with the standard sextupole correction scheme, provide the independent chromaticity control on the proton and antiproton helical orbits for both betatron planes. All four families are well decoupled so that the correction of one value has a little effect on the other.

*Work supported by the U.S. Department of Energy under contract No. DE-AC02-76CH03000....

"pmivanov@fnal.gov

\section{OCTUPOLE CUBIC NON-LINEARITY}

In this section we consider the betatron tune spread excited by the octupole cubic non-linearity for a Gaussian beam. The distribution function is expressed through the transverse actions $J_{x}$ and $J_{y}$ in the following form:

$$
f_{o}\left(J_{y}, J_{x}\right)=1 / \varepsilon_{x} \varepsilon_{y} \cdot \exp \left(-J_{y} / \varepsilon_{y}-J_{x} / \varepsilon_{x}\right) .
$$

Here $J_{x, y}=A_{x, y}^{2} / 2 \beta_{x, y}$ is $1 / 2$ of the single particle CourantSnyder invariant and $\varepsilon_{x, y}$ is the transverse rms-emittance. Detuning due to octupole non-linearity for particles with given betatron amplitudes $A_{x}, A_{y}$ is given by:

$$
\begin{aligned}
& \Delta v_{x}\left(J_{x}, J_{y}\right) \equiv v_{x}\left(J_{x}, J_{y}\right)-v_{x o}=\alpha_{x x} J_{x}-\alpha_{x y} J_{y}, \\
& \Delta v_{y}\left(J_{y}, J_{x}\right) \equiv v_{y}\left(J_{y}, J_{x}\right)-v_{y o}=\alpha_{y y} J_{y}-\alpha_{y x} J_{x} .
\end{aligned}
$$

Here the coefficients are determined by the octupole integral strengths, $K_{3}$, and the beta-functions, $\beta_{y, x}$ :

$$
\begin{aligned}
& \alpha_{y y, x x}=\frac{\partial v_{y, x}}{\partial J_{y, x}}=\frac{1}{8 \pi B \rho} \sum_{n, m}\left(\bar{K}_{3} \beta_{y, x}^{2}\right)_{n, m}, \\
& \alpha_{y x, x y}=-\frac{\partial v_{y}}{\partial J_{x}}=-\frac{\partial v_{x}}{\partial J_{y}}=\frac{1}{4 \pi B \rho} \sum_{n, m}\left(\bar{K}_{3} \beta_{x} \beta_{y}\right)_{n, m},
\end{aligned}
$$

and $n, m$ enumerate the octupoles and their families.

The betatron tune distribution function generated by the octupole cubic non-linearity is

$$
f\left(v_{y, x}\right)=\iint d J_{y} d J_{x} f_{o}\left(J_{y}, J_{x}\right) \cdot \delta\left(v_{y, x}-v_{y, x}\left(J_{y}, J_{x}\right)\right),
$$

where $\delta(v)$ is the Dirac delta function. Integration in Eq.(5) yields the expression for the tune distribution. For $\partial v_{y} / \partial J_{y}>0$ and $\partial v_{y} / \partial J_{x}<0$ one obtains:

$$
f\left(\Delta v_{y}\right)=\frac{1}{w}\left[\exp \left(\frac{-\Delta v_{y}}{\alpha_{y y} \varepsilon_{y}}\right) \Phi\left(\Delta v_{y}\right)+\exp \left(\frac{\Delta v_{y}}{\alpha_{x y} \varepsilon_{x}}\right) \Phi\left(-\Delta v_{y}\right)\right],
$$

where $\Phi(\geq 0)=1, \Phi(<0)=0$ is the Heaviside step function, and $w=\alpha_{y y} \varepsilon_{y}+\alpha_{x y} \varepsilon_{x}$ is the effective width of the betatron tune spread. Similarly, for $\partial v_{\boldsymbol{y}} / \partial \mathrm{J}_{\boldsymbol{y}}<0$ and $\partial v_{\boldsymbol{y}} / \partial \mathrm{J}_{\boldsymbol{x}}<0$, the tune distribution function is given by:

$$
f\left(\Delta v_{y}\right)=\frac{1}{w}\left[\exp \left(\frac{\Delta v_{y}}{\varepsilon_{x}\left|\alpha_{y x}\right|}\right)-\exp \left(\frac{\Delta v_{y}}{\varepsilon_{y}\left|\alpha_{y y}\right|}\right)\right] \Phi\left(-\Delta v_{y}\right) .
$$

\section{TUNE DISTRIBUTION FUNCTIONS}

The particle momentum spread makes an additional contribution to the betatron tune spread. Below we consider the case when linear chromaticities are equal to zero and, consequently, there are no synchro-betatron satellites. However, the quadratic chromaticity makes a non-zero contribution to the tune spread.

At injection and at the beginning of a store the proton longitudinal distribution in Tevatron is well approximated by the parabolic distribution function. Expressed through 
the single particle longitudinal emittance, $J_{S} \equiv \pi \Delta p \Delta \mathrm{z}=$ $\pi \eta \beta p_{0} \mathrm{c}\left\langle\left(\Delta p / p_{0}\right)^{2}\right\rangle / \Omega_{\mathrm{s}}$, it can be written as following,

$$
\rho\left(J_{s}\right)=2\left(1-J_{s} / 3 \varepsilon_{s}\right) / 3 \varepsilon_{s}, \quad J_{s}<3 \varepsilon_{s} .
$$

Here $\Delta p / p_{0}$ is the amplitude of the relative momentum deviation, $\eta$ is the slippage factor, $\Omega_{\mathbf{S}}$ is the synchrotron frequency, and the distribution is chosen so that $\left\langle J_{S}\right\rangle=\varepsilon_{S}$. The tune dependence on momentum is generated by the space charge and the quadratic chromaticity. The space charge tune shift for off-momentum particles is modulated with double synchrotron frequency resulting:

$$
v_{x, y}\left(J_{s}, t\right)=v_{o_{x, y}}-\Delta q_{x, y}\left(1-\left(J_{s} / 3 \varepsilon_{s}\right) \cos ^{2}\left(\Omega_{s} t\right),\right.
$$

where $\Delta q_{x, y}$ is the tune shift in the bunch center. For the Gaussian transverse and parabolic longitudinal distributions it is equal to:

$$
\Delta q_{x, y}=-\frac{3 N_{b} r_{p} R_{0}}{4 \sqrt{5} \beta^{2} \gamma^{3} \sigma_{s}}\left\langle\frac{\beta_{x, y}}{\sigma_{x, y}\left(\sigma_{x}+\sigma_{y}\right)}\right\rangle,
$$

where $\sigma_{x, y}(s)=\left(\varepsilon_{x, y} \beta_{x, y}(s)+D_{x, y}(s)^{2}{\sigma_{\Delta p / p}}^{2}\right)^{1 / 2}$, and $\langle.$.$\rangle denotes$ averaging over the machine circumference. Similar to the space charge the second order tune chromaticity, $\xi_{2(x, y)}$, introduces the betatron tune modulation:

$$
v_{x, y}\left(J_{s}, t\right)=v_{o(x, y)}+\xi_{2(x, y)}\left(J_{s} / 3 \varepsilon_{s}\right) \sin ^{2}\left(\Omega_{s} t\right),
$$

where we took into account that the linear chromaticities are equal to zero. Combining Eq. (9) with Eq. (11) and averaging over synchrotron motion we obtain the betatron tune shift for the off-momentum particles:

$$
\overline{\Delta v_{x, y}\left(J_{s}\right)}=\alpha_{s} J_{s} / 6 \varepsilon_{s}-\Delta q_{x, y},
$$

where $\alpha_{S}=6 \xi_{2(x, y)} \cdot \sigma_{\Delta p / p}{ }^{2}+\Delta q_{x, y}$ is the chromatic factor. Taking into account that the three-dimensional distribution can be factorized, $F_{o}\left(J_{x}, J_{y}, J_{s}\right)=f_{o}\left(J_{x}, J_{y}\right) \cdot \rho_{o}\left(J_{s}\right)$, the tune distribution function $F\left(\Delta v_{y}\right)$ is:

$$
F\left(\Delta v_{y}\right)=\int_{0}^{3 \varepsilon_{s}} f_{y}\left(\Delta v_{y}-\overline{\Delta v_{y}\left(J_{s}\right)}\right) \cdot \rho_{o}\left(J_{s}\right) d J_{s} .
$$

The integration in Eq. (13) yields the piecewise continuous function. For $\partial v_{y} / \partial J_{y}>0$ and $\partial v_{y} / \partial J_{x}<0$ it can be written in the following form:

$$
\begin{aligned}
& F\left(v_{y}\right)=F_{1} \cdot \Phi\left[v\left(v_{y}\right) / \alpha_{y y}\right]-F_{2} \cdot \Phi\left[-v\left(v_{y}\right) / \alpha_{y y}\right]- \\
& \quad-F_{3} \cdot \Phi\left[\left(2 v\left(v_{y}\right)-\alpha_{s y}\right) / \alpha_{y y}\right]+F_{4} \cdot \Phi\left[\left(\alpha_{s y}-2 v\left(v_{y}\right)\right) / \alpha_{y x}\right],
\end{aligned}
$$

where

$F_{1}=k_{y y}\left[\left(\alpha_{s y}+2 \varepsilon_{y} \alpha_{y y}\right)\left(1-\exp \left(-v\left(v_{y}\right) / \varepsilon_{y} \alpha_{y y}\right)\right)-2 v\left(v_{y}\right)\right]$, $F_{2}=k_{y x}\left[\left(\alpha_{s y}-2 \varepsilon_{x} \alpha_{y x}\right)\left(1-\exp \left(v\left(v_{y}\right) / \varepsilon_{x} \alpha_{y x}\right)\right)-2 v\left(v_{y}\right)\right]$, $F_{3}=k_{y y}\left[\alpha_{s y}+2 \varepsilon_{y} \alpha_{y y}\left(1-\exp \left(\left(\alpha_{s y}-2 v\left(v_{y}\right)\right) / 2 \varepsilon_{y} \alpha_{y y}\right)\right)-2 v\left(v_{y}\right)\right]$, $\left.F_{4}=k_{y x}\left[\alpha_{s y}-2 \varepsilon_{x} \alpha_{y x}\left(1-\exp \left(2 v\left(v_{y}\right)-\alpha_{s y}\right) / 2 \varepsilon_{x} \alpha_{y x}\right)\right)-2 v\left(v_{y}\right)\right]$, $v\left(v_{y}\right)=\Delta v_{y}+\Delta q_{y}$,

$k_{y y}=4 / \alpha_{s y}^{2}\left(1+\varepsilon_{x} \alpha_{y x} / \varepsilon_{y} \alpha_{y y}\right)$, $k_{y x}=4 / \alpha_{s y}^{2}\left(1+\varepsilon_{y} \alpha_{y y} / \varepsilon_{x} \alpha_{y x}\right)$.

In the experiments we examined two different polarities of the OF- and OD-octupole families. For both polarities the octupole settings were adjusted so that both vertical and horizontal modes would be Landau damped in the chromaticity range $-4 \leq \boldsymbol{\xi}_{x, y} \leq 4$ (see details in Ref [2]). The corresponding octupole settings are: $I_{O D}=4.2 A$,
$I_{O F}=5.5 A$ for set 1 , and $I_{O D}=6.0 A, I_{O F}=-4.5 A$ for set 2 . The corresponding tune distribution functions for $E=150$ $\mathrm{GeV}, \sigma_{\Delta \mathrm{p} / \mathrm{p}}=5.3 \cdot 10^{-4}$ and $\varepsilon_{x}=\varepsilon_{y}=1.7 \cdot 10^{-8} \mathrm{~m} \cdot \mathrm{rad}$ were calculated using Eq. (14) and are presented in Figures 1-2.

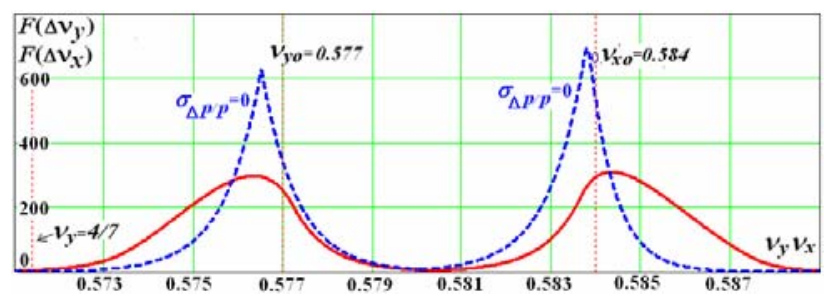

Fig.1. Tune distribution functions $F\left(\Delta v_{y}\right)$ and $F\left(\Delta v_{x}\right)$ for set 1 with $\xi_{2 y}=-4519, \xi_{2 x}=5597$ and $\xi_{1 x}=0, \xi_{1 y}=0$ (red solid lines). For comparison the dashed lines present the tune distributions for monochromatic beam.

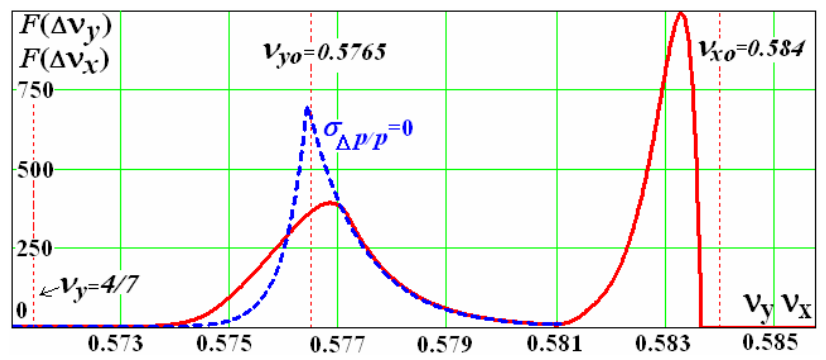

Fig.2. Tune distribution functions for octupole set 2 with $\xi_{2 y}=-3534$ and $\xi_{2 x}=-1653$ (details in the caption to Fig. 1).

\section{BEAM LIFETIME WITH OCTUPOLES}

There are two main mechanisms of particle loss at the injection energy. The first one is related to intra-beam scattering, which causes bunch lengthening and, consequently, particle losses from the RF bucket. The second one is related to particle losses due to betatron resonances $\left(v_{y}=4 / 7\right.$ and $\left.v_{x}=3 / 5\right)$ affecting particles in the tails of momentum distribution. As one can see from Figures 1-2, both the vertical and horizontal tune distributions for set 2 have smaller tails in direction of the dangerous resonances. Therefore, set 2 is preferable from the beam lifetime point of view. This improvement is related to the smaller value of the vertical quadratic chromaticity and a negative value for the horizontal quadratic chromaticity for set 2 . That causes tails to be created in the direction between the tunes where no strong betatron resonances are present.

Two definitions of the beam lifetime are used for the Tevatron. The first one, $\tau_{\mathrm{L}}$, determines the intensity decay in a single bunch; and the second one, $\tau_{T}$, determines the total intensity decay measured with a DC transformer. The second definition includes DC beam, i.e. the protons that left the RF buckets but stay in the beam. In the absence of transverse resonances, these particles can live in the machine a long time due to negligibly small energy losses at $E=150 \mathrm{GeV}$ and good vacuum. Measurements were performed for a normal proton intensity: 36 bunches with $N_{p}=2.5 \cdot 10^{11}$ per bunch, and no antiprotons. The results are presented in Table 1 . In the case of set 2 , the lifetime $\tau_{T}$ is mainly limited by vacuum conditions, thus verifying negligible effects of non-linear resonances on the beam lifetime. 
Table 1. Measured beam lifetimes.

\begin{tabular}{|l|c|c|}
\hline & $\tau_{L}$, hour & $\tau_{T}$, hour \\
\hline Octupole polarity set 1 & 25 & 42 \\
\hline Octupole polarity set 2 & 33 & 1400 \\
\hline Octupoles are turned off, $\boldsymbol{\xi}_{\boldsymbol{x}, \boldsymbol{y}} \cong 6$ & 11 & 36 \\
\hline
\end{tabular}

The introduction of octupoles with consecutive chromaticity zeroing resulted in decreasing of the chromatic tune modulation and significant lifetime improvement due to the suppression of the long-range beam-beam effects for both proton and antiproton beams during injection [3].

\section{OCTUPOLE EFFECTS AT ORBIT HELIXES}

\section{Linear betatron tune shift}

Linear betatron tune shifts (for particles with small oscillation amplitudes) due to the octupole fields at transition to the helical beam orbits are given by:

$$
\Delta v_{x, y}=\frac{ \pm 1}{8 \pi(B \rho)} \sum_{n, m}\left[\bar{K}_{3} \beta_{x, y}\left(\left(\Delta x-\Delta x_{o}\right)^{2}-\left(\Delta y-\Delta y_{o}\right)^{2}\right)\right]_{n, m} .
$$

Here $\Delta x, \Delta y$ are the orbit offsets at the octupole locations due to the beam separation, and $\Delta \boldsymbol{x}_{0}, \Delta \boldsymbol{y}_{0}$ are the octupole position errors with respect to the central orbit. Since $\Delta x, \Delta y>>\Delta x_{0}, \Delta y_{0}$ and $\Delta x, \Delta y$ have opposite values for proton and antiproton beams, the tune shifts are almost symmetric for both helixes:

$$
\Delta v_{x, y}(P) \approx \Delta v_{x, y}(\bar{P}) \propto \sum_{n, m}\left[\Delta x^{2}-\Delta y^{2}\right]_{n, m} .
$$

These tune shifts are compensated by the normal tune correction circuits at transition to the helical orbits. Small tune difference related to the orbit distortions is corrected by the corresponding feed-down sextupole circuits.

\section{Betatron linear coupling}

The equivalent skew-quadrupole strength of a particular octupole on the helical orbit is given by:

$$
\partial B_{y} / \partial y=\partial B_{x} / \partial x=-\left(\partial^{3} B_{y} / \partial x^{3}\right)\left(\Delta x-\Delta x_{o}\right)\left(\Delta y-\Delta y_{o}\right)
$$

At the ideal central orbit $\left(\Delta \boldsymbol{x}_{0}, \Delta \boldsymbol{y}_{0}=0\right)$, the product $\Delta \boldsymbol{x} \cdot \Delta \boldsymbol{y}$ has the same sign for the proton and antiproton orbits at the same octupole locations. Therefore, the change of the betatron coupling for both orbits is symmetric and can be easily compensated by regular skew-quadrupoles. The octupole families $\mathbf{O 1}$ and $\mathbf{O} 2$ do not produce any betatron coupling due to the alternating-sign of the lenses.

\section{Chromatic effects}

Octupole fields lead to an anti-symmetric change of linear chromaticities on the proton and antiproton helical orbits. The differential chromaticity is given by:

$$
\left.\Delta \xi_{1 x, y}=\frac{1}{4 \pi(B \rho)} \sum_{n, m} \bar{K}_{3} \beta_{x, y}\left(\left(\Delta x-\Delta x_{o}\right) D_{x}-\left(\Delta y-\Delta y_{o}\right) D_{y}\right)\right]_{n, m}
$$

It is seen that the central orbit offsets inside the octupoles cause a shift of the reference level for the differential chromaticity. It can be corrected by normal sextupoles.
A quadratic chromaticity change due to the octupole fields does not depend on the orbit displacements and can be evaluated in paraxial approximation as following:

$$
\Delta \xi_{2(x, y)}=\frac{1}{8 \pi(B \rho)} \sum_{n, m}\left[\bar{K}_{3} \beta_{x, y}\left(D_{x}^{2}-D_{y}^{2}\right)\right]_{n, m} .
$$

The octupole chromatic effects have been measured at the injection helixes as functions of the octupole currents (in Amps) for four independent families:

$\Delta \xi_{\mathrm{X}} \approx 4.7+4.08 \mathrm{I}_{\mathrm{O} 1}+0.70 \cdot \mathrm{I}_{\mathrm{O} 2}-0.31 \cdot \mathrm{I}_{\mathrm{OF}}-0.01 \cdot \mathrm{I}_{\mathrm{OD}}$

$\Delta \xi_{\mathrm{y}} \approx-1.2-1.76 \mathrm{I}_{\mathrm{O} 1}-3.10 \mathrm{I}_{\mathrm{O} 2}+0.18 \mathrm{I}_{\mathrm{OF}}+0.25 \mathrm{I}_{\mathrm{OD}}$

$\Delta \xi_{2 \mathrm{x}} \approx 1350-67 \cdot \mathrm{I}_{\mathrm{O} 1}+116 \cdot \mathrm{I}_{\mathrm{O} 2}+734 \cdot \mathrm{I}_{\mathrm{OF}}+50 \cdot \mathrm{I}_{\mathrm{OD}}$

$\Delta \xi_{2 \mathrm{y}} \approx-1759+5 \cdot \mathrm{I}_{\mathrm{O} 1}-102 \cdot \mathrm{I}_{\mathrm{O} 2}-175 \cdot \mathrm{I}_{\mathrm{OF}}-427 \cdot \mathrm{I}_{\mathrm{OD}}$

These measurements are in acceptable agreement with the computer simulation for the actual lattice model. Figure 3 shows the betatron tune dependence on the momentum difference $\delta_{\mathrm{p}}=\left(\mathrm{p}-\mathrm{p}_{0}\right) / \mathrm{p}_{0}$ for the Tevatron lattice with the OF-and OD-octupoles turned off and on. The residual lattice differential and quadratic chromaticities come from the Tevatron magnet imperfections.

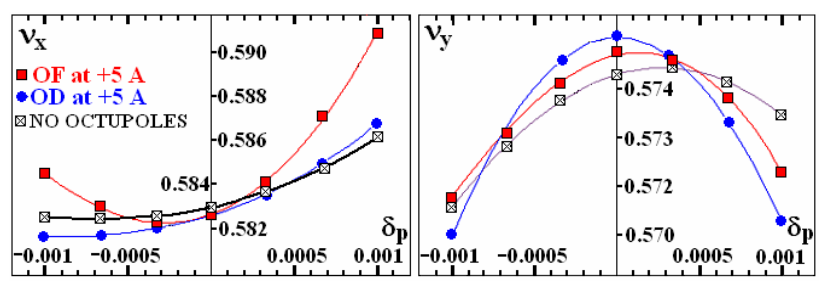

Fig.3. Dependence of tunes on the momentum difference for the Tevatron lattice on the central orbit.

\section{CONCLUSIONS}

It has been shown that the Tevatron can successfully operate with the considered scheme of octupole arrangement. Performed measurements are in good agreement with the analytical model and computer simulation. Because of operation at small chromaticities, the single beam lifetime is significantly better due to larger transverse dynamic aperture. The most important effect is the weakening of the long-range beam-beam interactions due to vanishing chromatic tune modulation at zero chromaticities. All undesirable octupole effects associated with the transition to the helical beam orbits have been compensated by existing Tevatron correction circuits.

\section{REFERENCES}

[1] H. Edwards, Ann. Rev. Nucl. Part. Sci., v. 35, pp. 605-660, 1985.

[2] P.M. Ivanov et al., "Landau Damping of the Weak Head-Tail Instability at Tevatron", PAC'05, MPPP042, these Proceedings.

[3] V. Shiltsev et al., "Beam-Beam Effects in the Tevatron RUN II", PAC'05, these Proceedings. 